\title{
Lower Respiratory Tract Pathogens and Their Antimicrobial Susceptibility Pattern: A 5-Year Study
}

\author{
Biagio Santella ${ }^{1,+} \oplus$, Enrica Serretiello ${ }^{1, \dagger}$, Anna De Filippis ${ }^{2}$, Veronica Folliero ${ }^{1}$, Domenico Iervolino ${ }^{3}$, \\ Federica Dell'Annunziata ${ }^{2}$, Roberta Manente ${ }^{1}$, Francesco Valitutti ${ }^{4} \oplus$, Emanuela Santoro ${ }^{5}$, Pasquale Pagliano ${ }^{5}$, \\ Massimiliano Galdiero ${ }^{1,2} \mathbb{C}$, Giovanni Boccia ${ }^{5,6, *(\mathbb{D})}$ and Gianluigi Franci $5,6, *(\mathbb{D})$
}

1 Section of Microbiology and Virology, University Hospital “Luigi Vanvitelli”, 80138 Naples, Italy; bi.santella@gmail.com (B.S.); enrica.serretiello@unicampania.it (E.S.); veronica.folliero@unicampania.it (V.F.); roberta.manente@studenti.unicampania.it (R.M.); massimiliano.galdiero@unicampania.it (M.G.)

2 Department of Experimental Medicine, University of Campania "Luigi Vanvitelli", 80138 Naples, Italy; anna.defilippis@unicampania.it (A.D.F.); federica.dellannunziata@unicampania.it (F.D.)

3 Department of Public Health and Infectious Diseases, Sapienza University of Rome, 00185 Rome, Italy; iervolino.1886704@studenti.uniroma1.it

4 Clinical Pediatrics and Pediatrics, University Hospital "San Giovanni di Dio e Ruggi d'Aragona", 84131 Salerno, Italy; francesco.valitutti@gmail.com

5 Department of Medicine, Surgery and Dentistry "Scuola Medica Salernitana", University of Salerno, 84081 Baronissi, Italy; esantoro@unisa.it (E.S.); ppagliano@unisa.it (P.P.)

6 Dai Dipartimento di Igiene Sanitaria e Medicina Valutativa U.O.C. Patologia Clinica E Microbiologica, Azienda Ospedaliero-Universitaria S. Giovanni di Dio e Ruggi D’Aragona Scuola Medica Salernitana,

check for updates

Citation: Santella, B.; Serretiello, E.; De Filippis, A.; Folliero, V.; Iervolino, D.; Dell'Annunziata, F.; Manente, R.; Valitutti, F.; Santoro, E.; Pagliano, P.; et al. Lower Respiratory Tract Pathogens and Their Antimicrobial Susceptibility Pattern: A 5-Year Study. Antibiotics 2021, 10, 851. https:// doi.org/10.3390/antibiotics10070851

Academic Editors: Albert Figueras and Seokhoon Jeong

Received: 21 May 2021

Accepted: 9 July 2021

Published: 13 July 2021

Publisher's Note: MDPI stays neutral with regard to jurisdictional claims in published maps and institutional affiliations.

\section{Copyright: (c) 2021 by the authors.} Licensee MDPI, Basel, Switzerland. This article is an open access article distributed under the terms and conditions of the Creative Commons Attribution (CC BY) license (https:// creativecommons.org/licenses/by/ $4.0 /)$.
Largo Città di Ippocrate, 84131 Salerno, Italy

* Correspondence: gboccia@unisa.it (G.B.); gfranci@unisa.it (G.F.)

+ These authors contributed equally to this work.

\begin{abstract}
Lower respiratory tract infections (LRTIs) are the most common infections in humans. It is estimated that 2.74 million deaths worldwide occur each year due to LRTIs. The aim of the study was to determine the frequency and antibiotic susceptibility pattern of microorganisms isolated from respiratory samples of patients with LRTIs. Between January 2015 and December 2019, a total of 7038 sputum and bronchoaspirate samples from suspected LRTI patients were collected. Among them, 2753 samples (39.1\%) showed significant microbial growth on culture media. The LRTI rate was higher in patients with male gender $(67.1 \%)$ and with age between $40-59$ years $(48.6 \%)$. The microorganism identification and antibiotic susceptibility testing were performed with Vitek 2 . Out of 4278 isolates species, 3102 (72.5\%) were Gram-negative bacteria, 1048 (24.5\%) were Gram-positive bacteria, and 128 (3.0\%) were Candida spp. Major microorganisms isolated were Acinetobacter baumannii (18.6\%), Staphylococcus aureus (15.2\%), Pseudomonas aeruginosa (14.2\%), and Klebsiella pneumoniae (10.9\%). In antimicrobial susceptibility testing, Staphylococcus aureus isolates were mostly resistant to Penicillin G $(84.1 \%)$ and Oxacillin $(48.1 \%)$, whereas they demonstrated maximum sensitivity to Tigecycline (100\%) and Linezolid (99.5\%). Among Gram-negative isolates, Acinetobacter baumannii showed maximum sensitivity to Colistin but was resistant to other antibiotics (95-99\%). Klebsiella pneumoniae isolates were mostly resistant to Cefotaxime (72.7\%) and sensitive to Gentamicin (54.3\%), and Pseudomonas aeruginosa was resistant to Ciprofloxacin (40.3\%) and sensitive to Amikacin (85.9\%). Gram-negative bacteria represented the species most commonly isolated. A high rate of antimicrobial resistance was observed in this study. In conclusion, the correct identification of causative microorganisms and their susceptibility patterns to antibiotics is crucial for choosing targeted and effective antibiotic therapy in LRTIs, and to prevent the emergence of multidrug-resistant bacteria.
\end{abstract}

Keywords: lower respiratory tract infections; antimicrobial resistance; epidemiology; nosocomial infections; antimicrobial stewardship 


\section{Introduction}

Lower respiratory tract infections (LRTIs) are the most common infections in humans. It is estimated that 2.74 million deaths worldwide occur each year due to LRTIs [1]. The commonest LRTIs are acute bronchitis, acute trachea bronchitis, chronic bronchitis, and pneumonia, which account for $4.4 \%$ of all hospital admissions and are associated with high morbidity, mortality, and excessive health costs [2-4]. The incidence and related mortality due to LRTIs can be influenced by several factors, including age, gender, season, the type of population at risk, but mainly to antibiotic therapy, the distribution of causative agents, and the prevalence of antimicrobial resistance $[5,6]$. The microbial aetiology of LRTIs and their susceptibility profile to antibiotics varies in different geographic regions $[7,8]$. The most common bacterial agents of LRTIs are Gram-positive bacteria such as Staphylococcus aureus and Enterococcus spp., and Gram-negative bacteria such as Pseudomonas spp., Acinetobacter spp., Klebsiella pneumoniae, and Haemophilus influenzae [9-12].

Due to the severity of these infections, there is an urgent need to adopt empirical antimicrobial treatment, before receiving the result on bacterial aetiology and antimicrobial susceptibility patterns $[13,14]$. Unfortunately, the ongoing spread of extended-spectrum $\beta$-lactamases and carbapenems has begun to limit the clinical effectiveness of $\beta$-lactam agents. This trend is presumably due to the empirical administration of antibacterial therapy $[15,16]$. The situation is further complicated by the emergence of multi-resistant pathogens, such as Klebsiella pneumoniae carbapenemase and Haemophilus influenzae $\beta$ lactamase $[17,18]$. Therefore, current knowledge of bacterial etiology and their antimicrobial susceptibility pattern would help to choose the antimicrobial therapy for bacterial LRTIs, to limit the development of antimicrobial resistance and reduce overall management costs [19-21]. The aim of this study was to describe the prevalence and patterns of antimicrobial sensitivity of microorganisms isolated from respiratory samples of patients with LRTIs, admitted to the San Giovanni di Dio e Ruggi d'Aragona Hospital (Salerno, Italy), to improve treatment protocols.

\section{Results}

\subsection{Incidence of LTRIs in Studied Patients}

From 2015 to 2019, a total of 7038 samples of sputum $(n=3113)$ and bronchoaspirate $(n=3925)$ were processed according to the standard microbiological methods. The LRTIs were confirmed by microscopic examination, with more than 25 leukocytes per field and with the presence of microorganisms. Out of them, 2753 samples $(39.1 \%)$ produced a significant growth of microorganisms on culture media (Table 1).

Table 1. Cases of LTRIs distributed by year.

\begin{tabular}{ccccccc}
\hline Samples & $\mathbf{2 0 1 5}$ & $\mathbf{2 0 1 6}$ & $\mathbf{2 0 1 7}$ & $\mathbf{2 0 1 8}$ & $\mathbf{2 0 1 9}$ & Total \\
\hline Positive bronchoaspirates & 404 & 409 & 341 & 402 & 420 & 1976 \\
Total bronchoaspirates & 597 & 617 & 752 & 939 & 1020 & 3925 \\
Positive sputum & 171 & 128 & 112 & 154 & 212 & 777 \\
Total sputum & 628 & 492 & 527 & 688 & 778 & 3113 \\
\hline
\end{tabular}

The average incidence of positive bronchoaspirate was $50.34 \%$, double that of positive sputum $24.96 \%(<0.01)$. About $2.5 \%$ of patients were in the age group of fewer than 19 years, $6.7 \%$ in $20-39$ years, $18.7 \%$ in $40-59$ years, $48.6 \%$ in $60-79$ years and $23.5 \%$ were of more than 80 years of age (Figure 1). Regarding gender, the LRTIs rate positive was higher in males than in females, $67.1 \%(1922)$ and $32.9 \%(831)$, respectively $(>0.01)$. 


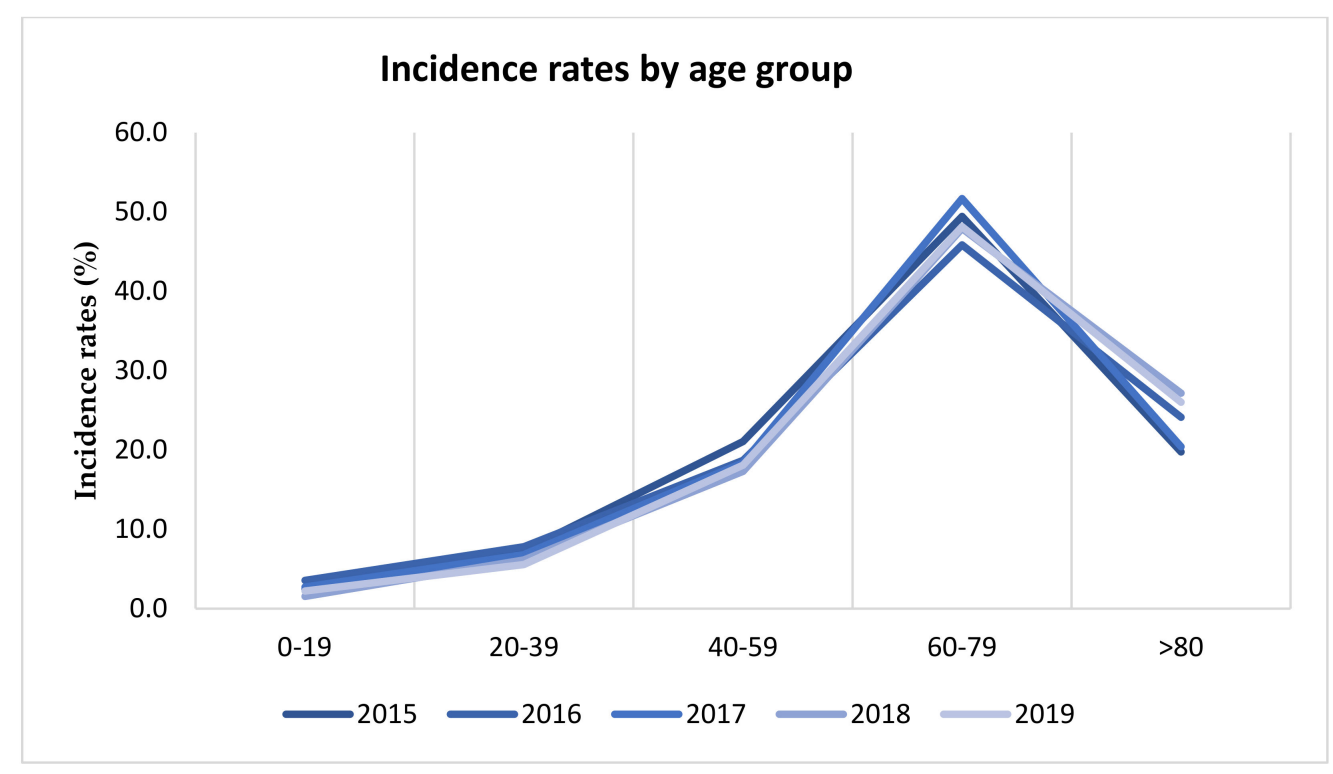

Figure 1. Distribution of positive cases by age group.

\subsection{Isolated Bacteria}

All pathogens identified over the five years of study, with respective incidence rates, were provided as additional data (Supplementary Table S1). Out of 2753 samples positive, 4278 species have been isolated and analyzed (Table 2).

Table 2. The number of species isolated per type of positive sample.

\begin{tabular}{ccccccc}
\hline Number Isolated & $\mathbf{2 0 1 5}$ & $\mathbf{2 0 1 6}$ & $\mathbf{2 0 1 7}$ & $\mathbf{2 0 1 8}$ & $\mathbf{2 0 1 9}$ & Total \\
\hline Broncoaspirate & 605 & 614 & 599 & 661 & 655 & 3134 \\
Sputum & 249 & 194 & 198 & 228 & 275 & 1144 \\
Total & 854 & 808 & 797 & 889 & 930 & 4278 \\
\hline
\end{tabular}

The rates of the number of isolates per positive sample are shown in Table 3, in $74.7 \%$ of cases there was monomicrobial growth, while in $25.1 \%$ there was significant polymicrobial growth. In the last year, only $15.2 \%$ of positive samples showed the growth of two microorganisms (Table 3).

Table 3. The rates of the number of isolates per positive sample.

\begin{tabular}{c|c|c|c}
\hline Number of Isolated Species per Sample & $\mathbf{1}$ & $\mathbf{2}$ & $\mathbf{3}$ \\
\hline $\mathbf{2 0 1 5}$ & 69.0 & 24.9 & 5.6 \\
$\mathbf{2 0 1 6}$ & 71.3 & 24.0 & 4.4 \\
$\mathbf{2 0 1 7}$ & 74.2 & 21.9 & 3.5 \\
$\mathbf{2 0 1 8}$ & 76.0 & 21.5 & 2.5 \\
$\mathbf{2 0 1 9}$ & 82.9 & 15.2 & 1.8 \\
\hline
\end{tabular}

Out of 4278 isolates identified, 3102 (72.5\%) were Gram-negative bacteria, 1048 (24.5\%) were Gram-positive bacteria, and 128 (3.0\%) were Candida spp. (Table 4).

Table 4. Subdivision of microorganisms isolated from positive samples expressed in percentage.

\begin{tabular}{cccccc}
\hline Microorganisms & $\mathbf{2 0 1 5}$ & $\mathbf{2 0 1 6}$ & $\mathbf{2 0 1 7}$ & $\mathbf{2 0 1 8}$ & $\mathbf{2 0 1 9}$ \\
\hline Gram positive & 23.3 & 25.5 & 25.1 & 23.7 & 25.0 \\
Gram negative & 73.5 & 71.7 & 72.0 & 73.5 & 71.8 \\
Fungi & 3.2 & 2.8 & 2.9 & 2.7 & 3.2 \\
\hline
\end{tabular}


Figure 2 shows the distribution of main microorganisms isolated from the LRTIs samples of hospitalized patients. The most common bacteria isolated were Acinetobacter baumannii, Pseudomonas aeruginosa, Staphylococcus aureus, and Klebsiella pneumoniae.

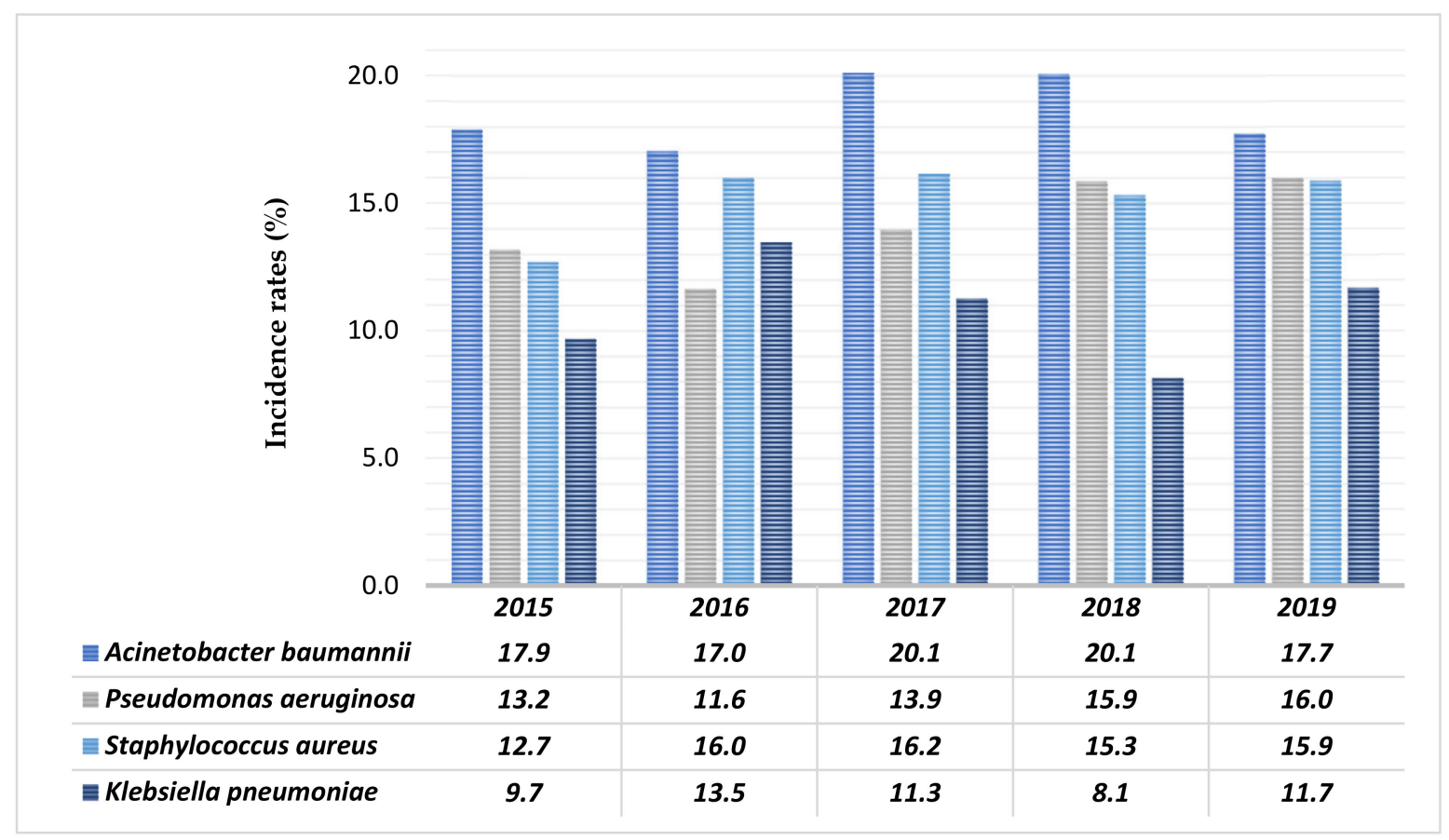

Figure 2. Annual incidence rates of the main bacteria isolated from positive samples.

\subsection{Prevalence of Antimicrobial Resistance among LRTIs Bacteria}

A very high rate of resistance (98-100\%) was observed among Acinetobacter baumannii isolates to Amoxicillin/Clavulanic acid, Cefotaxime, Ciprofloxacin, Ertapenem, Gentamicin, Imipenem, and Trimethoprim/Sulfamethoxazole. Only for Colistin, the isolate showed the maximum sensitivity with a rate of resistance less than $2 \%$ (Table 5).

Table 5. Antimicrobial resistance profile of Acinetobacter baumannii to antibiotics used in Hospital.

Acinetobacter baumannii

\begin{tabular}{|c|c|c|c|c|c|c|c|c|}
\hline Drug Class & Antibiotics & 2015 & 2016 & 2017 & 2018 & 2019 & * & $* *$ \\
\hline Penicillins & $\begin{array}{l}\text { Amoxicillin- } \\
\text { clavulanate }\end{array}$ & $100(148)$ & $100(129)$ & $100(127)$ & $100(148)$ & $100(135)$ & - & - \\
\hline Cephalosporins & Cefotaxime & $100(148)$ & $100(129)$ & $100(127)$ & $100(148)$ & $100(135)$ & - & - \\
\hline Fluoroquinolone & Ciprofloxacin & $98.0(148)$ & $95.3(129)$ & 94.5 (127) & $95.3(148)$ & $94.1(135)$ & 0.55 & - \\
\hline \multirow{2}{*}{ Carbapenems } & Ertapenem & $100(148)$ & $100(129)$ & $100(127)$ & $100(148)$ & $100(135)$ & - & - \\
\hline & Imipenem & $97.3(148)$ & $94.6(129)$ & 94.5 (127) & $94.2(148)$ & $96.3(135)$ & 0.68 & - \\
\hline $\begin{array}{l}\text { Aminoglycoside and } \\
\text { Tetracycline }\end{array}$ & Gentamicin & $94.6(148)$ & $94.6(129)$ & 85.8 (127) & $89.2(148)$ & $94.8(135)$ & 0.02 & 0.46 \\
\hline DHFR inhibitors & Trimethoprim/Sulfam. & $97.3(148)$ & $94.6(129)$ & 94.5 (127) & $94.2(148)$ & $96.3(135)$ & 0.41 & - \\
\hline Polypeptide & Colistin & $0(148)$ & $1.6(129)$ & $0(127)$ & $0(148)$ & $1.6(135)$ & 0.16 & - \\
\hline
\end{tabular}

${ }^{*} p$-value with chi-square, ${ }^{* *} p$-value with Cochran-Armitage trend test.

Among the Gram-negative, Pseudomonas aeruginosa was the second most frequently isolated species from positive samples. Against P. aeruginosa, Colistin was the drug that showed the lowest resistance rates, less than $9 \%$, despite the increasing trend during the 
study years, going from 3.8\% in 2015 to $5.7 \%$ in 2019 . However, Gentamicin showed a negative trend, with a decrease in resistance rate from $36.1 \%$ in 2015 to $12.3 \%$ in 2019 . A similar trend was shown for Amikacin with a decrease in resistance rate, less than ten percentage points in the last year (from 15.5\% to $4.9 \%$ ). Among the Carbapenems class, Meropenem showed lower resistance rates compared to Imipenem, with a difference of around ten percentage points. Furthermore, Imipenem shows a negative trend in resistance rates over the years, going from $44.9 \%$ in 2015 to $33.3 \%$ in 2019. Ciprofloxacin showed the highest resistance rates compared to the others. Ceftazidime, in the Cephalosporins class, showed high resistance rates in 2015 (44.4\%), higher than Cefepime (34.3\%), but in the last year, the resistance rate was less to Cefepime (22.1\% vs. $25.0 \%)$, due to negative trends. Moreover, Ciprofloxacin showed higher resistance rates than the other antibiotics described (Table 6).

Table 6. Antimicrobial resistance profile of Pseudomonas aeruginosa to antibiotics used in Hospital.

Pseudomonas aeruginosa

\begin{tabular}{|c|c|c|c|c|c|c|c|c|}
\hline Drug Class & Antibiotics & 2015 & 2016 & 2017 & 2018 & 2019 & * & $* *$ \\
\hline Penicillins & Piperacillin/Tazobactam & $47.2(106)$ & $40.0(85)$ & $28.0(50)$ & $33.9(112)$ & $33.9(121)$ & 0.10 & - \\
\hline \multirow{2}{*}{ Cephalosporins } & Ceftazidime & $44.4(108)$ & $39.8(88)$ & $51.2(86)$ & $27.0(115)$ & $22.1(122)$ & $<0.01$ & $<0.01$ \\
\hline & Cefepime & $34.3(108)$ & $28.4(88)$ & $37.1(89)$ & $23.3(90)$ & $25.0(120)$ & 0.16 & - \\
\hline Fluoroquinolones & Ciprofloxacin & $51.8(108)$ & $35.2(88)$ & $45.3(86)$ & $27.6(116)$ & $41.8(122)$ & 0.03 & 0.05 \\
\hline \multirow{2}{*}{ Carbapenems } & Imipenem & 44.9 (107) & $47.7(88)$ & $39.5(86)$ & $39.3(89)$ & $33.3(120)$ & 0.25 & - \\
\hline & Meropenem & $27.8(108)$ & $31.8(88)$ & $28.2(85)$ & $20.9(115)$ & $23.1(121)$ & 0.40 & - \\
\hline \multirow{2}{*}{$\begin{array}{l}\text { Aminoglycosides and } \\
\text { Tetracycline }\end{array}$} & Gentamicin & $36.1(108)$ & $25.0(88)$ & $37.2(86)$ & $10.3(116)$ & $12.3(122)$ & $<0.01$ & $<0.01$ \\
\hline & Amikacin & $15.5(103)$ & $18.4(87)$ & $24.7(85)$ & $6.9(116)$ & $4.9(122)$ & $<0.01$ & $<0.01$ \\
\hline Polypeptide & Colistin & $3.8(104)$ & $2.5(81)$ & $0(63)$ & $8.3(84)$ & 5.7 (87) & 0.12 & - \\
\hline
\end{tabular}

Among the Gram-positive bacteria isolated, the most frequent species was Staphylococcus aureus. Resistance rates of S. aureus to Penicillin G were above 83\% in 2016-2019, higher than in 2015. Moreover, to Oxacillin, S. aureus showed an increasing resistance trend from 2015 to 2019 , going from $32.4 \%$ to $60.7 \%$. Similar results were showed for the Macrolide class, in which Erythromycin showed higher resistance rates in 2019 (51.9\%). This antibiotic class, showed a similar trend, with a higher resistance rate in 2017. Moreover, Clindamycin has observed a positive trend, with an increase in the resistance rate, more than ten percentage points, from 2015 to 2019. The rate of resistance to Levofloxacin, showed a positive trend, with different resistance rates, and an increase to more than twenty percentual points in confront to the first year of study, from $28.1 \%$ in 2015 to $54.9 \%$ in 2019. The Aminoglycosides and Tetracyclines class showed resistance rates much lower than the previously listed classes, showing maximum sensibility to Tigecycline, with resistance rates to $0 \%$. Glycopeptides class showed similar results, in which, Vancomycin and Teicoplanin showed very low resistance rates, but a worrying increasing trend over the years of study, going from $0 \%$ in 2015 to $4.2 \%$ and $2.5 \%$ in 2019, respectively. Finally, for Linezolid, resistance rates better than the previous ones have been recorded, with a percentage of less than $2.0 \%$ from 2015 to 2019 (Table 7).

Klebsiella pneumoniae was the third most frequently isolated Gram-negative species, with a mean percentage of $10.9 \%$. Against K. pneumoniae, the tested antibiotics belonging to the Penicillin and Cephalosporin class showed similar high rates of resistance, exceeding 70\%, including Ciprofloxacin. However, Amoxicillin-clavulanate and Cefepime have shown decreasing resistance rates over the years, with a difference of more than twenty percentage points over the past year. Moreover, the Carbapenem class showed high resistance rates with the highest values exceeding 70\%. Resistance values lower than 
those discussed previously, around 50\%, are highlighted by the class of Aminoglycosides and Tetracyclines. Finally, Colistin showed the lowest resistance rates compared to the other antibiotics, around 15\%, ranking it as the most efficient antibiotic against this species (Table 8).

Table 7. Antimicrobial resistance profile of Staphylococcus aureus to antibiotics used in Hospital.

\section{Staphylococcus aureus}

\begin{tabular}{|c|c|c|c|c|c|c|c|c|}
\hline Drug Class & Antibiotics & 2015 & 2016 & 2017 & 2018 & 2019 & * & $* *$ \\
\hline \multirow{2}{*}{ Penicillins } & Penicillin G & $78.1(105)$ & 83.5 (121) & $89.3(103)$ & $86.1(101)$ & $83.3(120)$ & 0.12 & - \\
\hline & Oxacillin & $32.4(105)$ & $35.5(121)$ & $54.4(103)$ & $56.4(101)$ & $60.7(112)$ & $<0.01$ & $<0.01$ \\
\hline \multirow{3}{*}{ Macrolides } & Erythromycin & $40.0(105)$ & $46.3(121)$ & 59.7 (103) & $45.0(100)$ & $51.8(112)$ & 0.06 & - \\
\hline & Azithromycin & $38.5(96)$ & $45.1(102)$ & $59.2(103)$ & $44.3(70)$ & NA & 0.03 & 0.10 \\
\hline & Clarithromycin & $38.5(96)$ & $45.1(102)$ & $59.2(103)$ & $44.3(70)$ & NA & 0.03 & 0.10 \\
\hline Lincosamides & Clindamycin & $36.3(102)$ & 40.7 (118) & $52.4(103)$ & 40.7 (113) & $50.0(112)$ & 0.09 & - \\
\hline Fluoroquinolones & Levofloxacin & $28.1(96)$ & $40.2(102)$ & $58.3(103)$ & $45.5(101)$ & $54.9(113)$ & $<0.01$ & $<0.01$ \\
\hline \multirow{3}{*}{$\begin{array}{l}\text { Aminoglycosides and } \\
\text { Tetracycline }\end{array}$} & Gentamicin & $8.6(105)$ & $12.4(121)$ & $2.9(103)$ & $4.0(101)$ & $5.4(112)$ & 0.03 & 0.05 \\
\hline & Tetracycline & $8.6(105)$ & $2.5(121)$ & $2.9(103)$ & $4.0(101)$ & $5.8(120)$ & 0.17 & - \\
\hline & Tigecycline & $0(96)$ & $0(113)$ & $0(103)$ & $0(113)$ & $0(121)$ & - & - \\
\hline \multirow{2}{*}{ Glycopeptides } & Teicoplanin & $0(105)$ & $0(102)$ & $1.0(103)$ & 0 (113) & $4.2(118)$ & $<0.01$ & $<0.01$ \\
\hline & Vancomycin & $0(96)$ & $0(121)$ & $1.0(103)$ & $0(113)$ & $2.5(120)$ & 0.10 & - \\
\hline Oxazolidinones & Linezolid & $0(105)$ & $1.7(121)$ & $0(103)$ & $0(113)$ & $0.8(121)$ & 0.31 & - \\
\hline
\end{tabular}

${ }^{*} p$-value with chi-square, ${ }^{* *} p$-value with Cochran-Armitage trend test.

Table 8. Antimicrobial resistance profile of Klebsiella pneumoniae to antibiotics used in hospital.

Klebsiella pneumoniae

\begin{tabular}{|c|c|c|c|c|c|c|c|c|}
\hline Drug Class & Antibiotics & 2015 & 2016 & 2017 & 2018 & 2019 & * & $* *$ \\
\hline \multirow{2}{*}{ Penicillins } & Amoxicillin-clavulanate & $78.3(83)$ & $75.8(91)$ & $57.8(71)$ & $57.6(59)$ & $59.3(81)$ & $<0.01$ & $<0.01$ \\
\hline & Piperacillin/Tazobactam & $77.6(85)$ & $80.4(102)$ & $66.2(71)$ & $65.0(60)$ & $77.5(89)$ & 0.09 & - \\
\hline \multirow{3}{*}{ Cephalosporins } & Cefepime & $74.2(85)$ & $77.4(102)$ & $57.7(71)$ & $57.7(59)$ & $44.9(91)$ & $<0.01$ & 0.01 \\
\hline & Cefotaxime & $81.2(80)$ & $77.4(102)$ & $66.2(71)$ & $56.7(60)$ & $82.0(89)$ & $<0.01$ & 0.10 \\
\hline & Ceftazidime & $75.3(85)$ & $78.4(102)$ & $66.2(71)$ & $57.6(59)$ & $82.0(89)$ & $<0.01$ & $<0.03$ \\
\hline Fluoroquinolones & Ciprofloxacin & $76.5(85)$ & $77.4(102)$ & $64.8(71)$ & $53.3(60)$ & $82.0(89)$ & $<0.01$ & 0.24 \\
\hline \multirow{2}{*}{ Carbapenems } & Ertapenem & $57.6(59)$ & $72.5(102)$ & $47.9(71)$ & $46.7(60)$ & $70.8(89)$ & $<0.01$ & $<0.01$ \\
\hline & Meropenem & $70.2(84)$ & $72.5(102)$ & $48.6(70)$ & $45.8(59)$ & $71.6(88)$ & $<0.01$ & 0.23 \\
\hline \multirow{2}{*}{$\begin{array}{l}\text { Aminoglycosides and } \\
\text { Tetracycline }\end{array}$} & Gentamicin & $45.9(85)$ & $49.0(102)$ & $52.1(71)$ & $36.7(60)$ & $44.9(89)$ & 0.47 & 0.50 \\
\hline & Tigecycline & $53.1(64)$ & $64.4(101)$ & $52.1(71)$ & $44.1(59)$ & $59.1(88)$ & 0.12 & - \\
\hline DHFR inhibitors & Trimethoprim/Sulfam. & $74.1(85)$ & $72.5(102)$ & $63.4(71)$ & $41.7(60)$ & $34.8(89)$ & $<0.01$ & $<0.01$ \\
\hline Polypeptide & Colistin & $33.7(83)$ & $28.3(99)$ & $11.1(54)$ & $2.3(39)$ & $42.5(73)$ & $<0.01$ & $<0.01$ \\
\hline
\end{tabular}

${ }^{*} p$-value with chi-square, ${ }^{* *} p$-value with Cochran-Armitage trend test.

\section{Discussion}

The aim of the study was to determine the prevalence of microorganisms responsible to LRTIs in our hospital and their susceptibility profile to antibiotics. From 2015 to 2019, 7038 sputum and bronchoaspirate samples were analyzed, among them 2753 (39.1\%) produced a significant growth of microorganisms on culture media, with a higher incidence 
in bronchoaspirate samples compared to sputum (52.7\% vs. $25.1 \%)$. Recently published articles show similar incidence rates to ours [22-24].

LRTIs were more common in males than females (67.1\% vs. $32.9 \%)$. Male prevalence of LRTI may be due to some associated risk factors for respiratory tract infection such as smoking, alcohol consumption and COPD [12,25]. Age range distribution showed that the incidence of LTRIs increases rapidly with increasing age, with a maximum incidence between 60 and 79 years. Among 4278 pathogenic microorganism isolates, $72.5 \%$ were Gram-negative bacteria, 24.5\% were Gram-positive bacteria, and 3.0\% were Candida spp. In similar studies, Gram-negative bacteria represented the species most commonly isolated from samples by patients of lower respiratory tract infections [26,27]. In the current investigation, monomicrobial growth was found in $74.7 \%$ of the cases, whereas $25.3 \%$ were polymicrobial, and identification of the polymicrobial infection is very important for treatment strategies. In another study, monomicrobial growth was found in $80 \%$ of cases, whereas $20 \%$ were polymicrobial [28]. The major microorganisms causing LRTI were Acinetobacter baumannii, Staphylococcus aureus, Pseudomonas aeruginosa, and Klebsiella pneumoniae. This observation is similar to other studies, such that of Christopher Aye Egbe et al., in which the most frequent mixed infection was caused by Klebsiella spp. and Pseudomonas spp. [12,24,29].

Antimicrobial susceptibility test performed on Acinetobacter baumannii isolates showed that Colistin was the most effective (nearly 100\% sensitivity) and Amoxicillin/Clavulanic acid, Cefotaxime, Oxacillin, Ciprofloxacin, Ertapenem, Gentamicin, Imipenem, and Trimethoprim/Sulfamethoxazole were the least effective ones (98-100\% resistance). The causes may be due to the high propensity of this species to easily acquire resistance genes and the ability to persist and multiply in a hospital setting. The high rates of resistance to first-line antimicrobial drugs shown by A. baumannii isolate highlight the need to find new effective molecules to counter this threat [30-34].

Pseudomonas aeruginosa was the second commonest organism among Gram-negative bacteria isolated. In the case of P. aeruginosa, Gentamycin, Amikacin, and Colistin were the most effective antibiotics (less to $20 \%$ resistance), contrary to Piperacillin/Tazobactam and Ciprofloxacin that was the least effective ( $40-50 \%$ resistance). The antibiotic resistance rates shown in P. aeruginosa are similar to results shown by a study by Yayan J. et al. [35]

The most species isolated of Gram-positive was Staphylococcus aureus. This species was found to be mostly resistant against Penicillin G and medium resistant to Oxacillin, followed by Macrolides, Lincosamides and Fluoroquinolones class. The antibiotics more efficient against $S$. aureus were Gentamicin, Tetracycline, Tigecycline, Teicoplanin, Vancomycin, and Linezolid, with a rate of resistance less to $10 \%$. Oxacillin, Vancomycin, and Teicoplanin showed very low resistance rates, but a worrying increasing trend over the years of study. This event may have been caused by the increase in Oxacillin-resistant S. aureus MRSA, which are treated with Vancomycin and Teicoplanin; consequently, an increase in resistance to the latter has been observed. The same event has been documented in other articles [36-40].

Among Gram-negative bacteria, in terms of frequency, P. aeruginosa was followed by Klebsiella pneumoniae. This species was mostly resistant to Penicillin, Cephalosporins, and Fluoroquinolones class (above 70\%). Moreover, in this case, the most efficient antibiotic was Colistin, with resistance rates higher than 10\%, while the Carbapenems, Aminoglycosides and Tetracyclines class have higher resistances, around $45 \%$. In a study by Ahmed et al., K. pneumoniae showed similar resistance rates, except for Piperacillin/Tazobactam, with a reported resistance rate of $18.2 \%$, lower than that shown in our study $(73.3 \%)$ [41]. This difference could be due to a different empirical antimicrobial treatment and a different geographic region, as described by Prestinaci et al. [42]. Very recent reports indicated Gram-negative bacteria were the major microorganisms involved in LRTIs $[12,43]$ and were reported to increase resistance to Carbapenems and Fluoroquinolones for K. pneumoniae. Another hand, among Gram-positive, S. aureus was the main bacteria isolated reported and showed high susceptibility to Vancomycin and Linezolid, findings are the same as 
observed in our study. According to a recent WHO report on the epidemiology of infectious diseases, LRTI tops the list in developing countries [44]. This study reveals the incidence of the main pathogens responsible for LRTI and their resistance to the most commonly used antibiotics in hospital settings $[45,46]$. The results show high rates of resistance by the most commonly isolated bacteria. Resistance to Fluoroquinolones and 3rd generation Cephalosporins is rapidly emerging [47]. This may be due to irrational drug abuse and the resulting mutation of pathogenic microorganisms [48,49].

\section{Materials and Methods}

\subsection{Samples Collection}

The present retrospective study was conducted in the Microbiology department of University Hospital "San Giovanni di Dio e Ruggi d'Aragona" in the period between January 2015 and December 2019.

Sputum and bronchial aspiration samples from patients with suspected lower respiratory tract infection (LRTI) were analyzed. A total of 7038 samples were collected from patients of all age and gender groups.

\subsection{Identification and Antimicrobial Susceptibility Testing}

The samples were collected in sterile containers and immediately transported to the bacteriology laboratory and were processed further. Only those samples with an adequate amount of sputum were accepted. Those that contained an inadequate amount of sputum for analysis or that contained only saliva were excluded.

Sputum specimen for bacteriological culture was subjected to Gram-staining and examined microscopically. In microscopic examination sputum smear containing less than 10 squamous epithelial cells and more than 25 leucocytes or pus cells per low power, field confirmed the reliability of the specimen, indicating that it was not contaminated with saliva. The samples of sputum which were very thick and mucoid were first homogenized with commercially available sputasol containing $0.01 \%$ dithiothreitol and were incubated at $37^{\circ} \mathrm{C}$ for $30 \mathrm{~min}$ for complete homogenization of sputum.

All samples were immediately plated on Chocolate agar, blood agar, MacConkey, and Sabouraud Glucose agar medium (Oxoid, Hampshire, UK), and were incubated at $37^{\circ} \mathrm{C}$. The Chocolate agar was incubated at $37^{\circ} \mathrm{C}$ in a $5 \% \mathrm{CO}_{2}$ atmosphere. After $18-36 \mathrm{~h}$ of incubation, each plate was examined, and bacterial identification and antimicrobial susceptibility test were performed.

Sputum or bronchoaspirate showing less than $10^{4} \mathrm{CFU} / \mathrm{ml}$ by semiquantitative culture were regarded as commensal or contaminant and were excluded. The bacterial identification and antimicrobial susceptibility test were performed utilizing a Vitek 2 (bioMerieux, Marcy l'Etoile, France), using an identification card (ID-GN for Gram-Negative, ID-GP for Gram-positive, YST for yeast) and the AST-659 (for Staphylococci), AST-658 (for Enterococci and S. agalactiae), AST-ST03 (for Pneumococci), AST-379 (for Enterobacteriaceae), and AST-397 (for GN non-fermenters) susceptibility cards, according to the manufacturer's instructions. The results of antimicrobial susceptibility were interpreted as "susceptible", "resistant", or "intermediate" according to EUCAST guidelines. The Quality Control process encompasses the annual service and certification of the instrument by bioMerieux and the Quality Control of each lot of Gram-negative (GN), Gram-positive (GP) cards using four strains: Enterococcus ATCC 700,327 and Staphylococcus aureus ATCC 29,213 for GP; Enterobacter ATCC 700,323 and Klebsiella oxytoca ATCC 700,324 for GN.

The following antibiotics were included in the present study: amikacin (AMK), amoxicillin/clavulanic acid (AMC), azithromycin (AZM), cefepime (FEP), cefotaxime (CTX), ceftazidime (CAZ), ciprofloxacin (CIP), clarithromycin (CLR), colistin (COL), ertapenem (ETP), erythromycin (ERY), gentamicin (GEN), imipenem (IPM), levofloxacin (LVX), linezolid (LNZ), meropenem (MEM), moxifloxacin (MFX), oxacillin (OXA), piperacillin/tazobactam (TZP), teicoplanin (TEC), tigecycline (TCG), vancomycin (VAN). 


\subsection{Ethical Consideration Statement}

Ethical approval by the Human Research Ethics Committee was not requested. The present study used laboratory management data, collected from a database. This is a retrospective study and not directly associated with patients.

\subsection{Statistical Analysis}

Demographic data of patients, including age, gender, isolated strain(s), and drug sensitivity results, were used for the analysis. The crude incidence and age- and sexstandardized incidence were calculated. The chi-framework test was used to compare the differences in the incidence of bacteria in hospitalized patients and the differences among antibiotic sensitivities over the range of years considered in the study. A chi-square test was used to verify the possible associations between the categorical variables, while the Cochran-Armitage trend test was used to verify the existence of a trend, the existence of a trend was checked only for antibiotics that showed statistically significant differences in the distribution of resistance during the years under consideration, an alpha equal to $5 \%$ was considered for both tests, therefore those associations that had a $p$-value $<0.05$ were considered statistically significant. The IBM Statistical Package for Social Sciences Version 22.00 (SPSS Inc., Chicago, IL, USA) was used for data analysis.

\section{Conclusions}

Bacterial aetiology varies in different regions and populations. Therefore, infection surveillance studies have become increasingly necessary and important to control LRTIs. The correct identification of pathogenic microorganisms and their susceptibility patterns to antibiotics can be useful for our healthcare professionals to choose the most targeted and effective antibiotic therapy. Furthermore, this study could represent an alarm for the competent authorities to develop effective policies on the precise and rational prescription of antibiotics. However, our research has certain limitations, partly because it was only focused on LRTI patients admitted to the San Giovanni di Dio e Ruggi d'Aragona Hospital. Therefore, multicenter, longitudinal, prospective research is required to confirm our findings. However, molecular diagnostic tests will need to be strengthened for faster results. All this could reduce the phenomenon of antibiotic resistance and ensure maximum safety for the health of patients.

Supplementary Materials: The following are available online at https://www.mdpi.com/article/ 10.3390/antibiotics10070851/s1, Table S1: Percentage trend of all bacterial isolates that caused LTRIs infections.

Author Contributions: Conceptualization, B.S.; writing—review and editing, B.S.; supervision, A.D.F., G.F., V.F. and M.G.; funding acquisition, G.B. and G.F.; data curation, B.S. and D.I.; visualization, E.S. (Enrica Serretiello), F.D., R.M., F.V., E.S. (Emanuela Santoro) and P.P. All authors have read and agreed to the published version of the manuscript.

Funding: This research received no external funding.

Institutional Review Board Statement: Ethical approval by the Human Research Ethics Committee was not requested for this study. The resignation was given as our study used laboratory management data and clinical information on patients, collected from databases. This is a retrospective study and not directly associated with patients. This study was consistent with the principles of the Helsinki Declaration.

Informed Consent Statement: Patient consent in this study was not necessary because our study used laboratory management data and clinical information on patients, collected from databases. Moreover, was maintaining the patient incognito.

Data Availability Statement: Epidemiological data used to support the results of this study are included in the article. 
Acknowledgments: The authors would like to thank the staff of the U.O.C. University Hospital of Campania "Luigi Vanvitelli" in Naples, Italy and the staff of University Hospital "San Giovanni di Dio e Ruggi d'Aragona", for their contributions.

Conflicts of Interest: The authors declare no conflict of interest.

\section{References}

1. Collaborators, G.L. Estimates of the global, regional, and national morbidity, mortality, and aetiologies of lower respiratory tract infections in 195 countries: A systematic analysis for the Global Burden of Disease Study 2015. Lancet Infect. Dis. 2017, 17, 1133-1161. [CrossRef]

2. Nowicki, J.; Murray, M.T. Bronchitis and Pneumonia. Textbook of Natural Medicine; Elsevier: Amsterdam, The Netherlands, 2020; pp. 1196-1201.e1. [CrossRef]

3. Ortqvist, A. Treatment of community-acquired lower respiratory tract infections in adults. Eur. Respir. J. Suppl. 2002, 36, 40s-53s. [CrossRef]

4. Fair, R.J.; Tor, Y. Antibiotics and bacterial resistance in the 21st century. Perspect. Med. Chem. 2014, 6, 25-64. [CrossRef] [PubMed]

5. Millett, E.R.C.; Quint, J.K.; Smeeth, L.; Daniel, R.M.; Thomas, S.L. Incidence of community-acquired lower respiratory tract infections and pneumonia among older adults in the United Kingdom: A population-based study. PLoS ONE 2013, 8, e75131. [CrossRef] [PubMed]

6. Torres, A.; Cilloniz, C.; Niederman, M.S.; Menéndez, R.; Chalmers, J.D.; Wunderink, R.G.; van der Poll, T. Pneumonia. Nat. Rev. Dis. Primers 2021, 7, 25. [CrossRef]

7. Ahmed, S.M.A.-Z.; Abdelrahman, S.S.; Saad, D.M.; Osman, I.S.; Osman, M.G.; Khalil, E.A.G. Etiological Trends and Patterns of Antimicrobial Resistance in Respiratory Infections. Open Microbiol. J. 2018, 12, 34-40. [CrossRef]

8. Santella, B.; Folliero, V.; Pirofalo, G.M.; Serretiello, E.; Zannella, C.; Moccia, G.; Santoro, E.; Sanna, G.; Motta, O.; De Caro, F. Sepsis-A Retrospective Cohort Study of Bloodstream Infections. Antibiotics 2020, 9, 851. [CrossRef] [PubMed]

9. Gebre, A.B.; Begashaw, T.A.; Ormago, M.D. Bacterial profile and drug susceptibility among adult patients with community acquired lower respiratory tract infection at tertiary hospital, Southern Ethiopia. BMC Infect. Dis. 2021, 21, 440. [CrossRef] [PubMed]

10. Mishra, S.; Kattel, H.; Acharya, J.; Shah, N.; Shah, A.; Sherchand, J.; Rijal, B.; Pokhrel, B. Recent trend of bacterial aetiology of lower respiratory tract infection in a tertiary care centre of Nepal. Int. J. Infect. Microbiol. 2012, 1, 3-8. [CrossRef]

11. Behera, B.; Sahu, K.; Bhoi, P.; Mohanty, J. Prevalence and antimicrobial susceptibility patterns of bacteria in ICU patients with lower respiratory tract infection: A cross-sectional study. J. Acute Dis. 2020, 9, 157-160. [CrossRef]

12. Singh, S.; Sharma, A.; Nag, V. Bacterial pathogens from lower respiratory tract infections: A study from Western Rajasthan. J. Fam. Med. Prim. Care 2020, 9, 1407-1412. [CrossRef]

13. Majumder, M.A.A.; Rahman, S.; Cohall, D.; Bharatha, A.; Singh, K.; Haque, M.; Gittens-St Hilaire, M. Antimicrobial stewardship: Fighting antimicrobial resistance and protecting global public health. Infect. Drug Resist. 2020, 13, 4713. [CrossRef]

14. Metersky, M.L.; Masterton, R.G.; Lode, H.; File, T.M.; Babinchak, T. Epidemiology, microbiology, and treatment considerations for bacterial pneumonia complicating influenza. Int. J. Infect. Dis. 2012, 16, e321-e331. [CrossRef]

15. Aslan, A.T.; Akova, M. Extended spectrum $\beta$-lactamase producing enterobacteriaceae: Carbapenem sparing options. Expert Rev. Anti Infect. 2019, 17, 969-981. [CrossRef]

16. Paterson, D.L.; Bonomo, R.A. Extended-spectrum beta-lactamases: A clinical update. Clin. Microbiol. Rev. 2005, 18, 657-686. [CrossRef]

17. Ferreira, R.L.; da Silva, B.C.M.; Rezende, G.S.; Nakamura-Silva, R.; Pitondo-Silva, A.; Campanini, E.B.; Brito, M.C.A.; da Silva, E.M.L.; Freire, C.C.d.M.; da Cunha, A.F.; et al. High Prevalence of Multidrug-Resistant Klebsiella pneumoniae Harboring Several Virulence and $\beta$-Lactamase Encoding Genes in a Brazilian Intensive Care Unit. Front. Microbiol. 2019, 9, 3198. [CrossRef] [PubMed]

18. Tristram, S.; Jacobs, M.R.; Appelbaum, P.C. Antimicrobial resistance in Haemophilus influenzae. Clin. Microbiol. Rev. 2007, 20, 368-389. [CrossRef] [PubMed]

19. Llor, C.; Bjerrum, L. Antimicrobial resistance: Risk associated with antibiotic overuse and initiatives to reduce the problem. Ther. Adv. Drug Saf. 2014, 5, 229-241. [CrossRef]

20. Esposito, S.; De Simone, G.; Gioia, R.; Noviello, S.; Pagliara, D.; Campitiello, N.; Rubino, C.; Lo Pardo, D.; Boccia, G.; De Caro, F.; et al. Deep tissue biopsy vs. superficial swab culture, including microbial loading determination, in the microbiological assessment of Skin and Soft Tissue Infections (SSTIs). J. Chemother. 2017, 29, 154-158. [CrossRef] [PubMed]

21. Brusaferro, S.; Arnoldo, L.; Finzi, G.; Mura, I.; Auxilia, F.; Pasquarella, C.; Agodi, A.; Pitzurra, M.; Arrigoni, C.; Barchitta, M.; et al. Hospital hygiene and infection prevention and control in Italy: State of the art and perspectives. Ann. Ig. 2018, 30, 1-6. [CrossRef] [PubMed]

22. Duan, N.; Du, J.; Huang, C.; Li, H. Microbial Distribution and Antibiotic Susceptibility of Lower Respiratory Tract Infections Patients From Pediatric Ward, Adult Respiratory Ward, and Respiratory Intensive Care Unit. Front. Microbiol. 2020, 11, 1480. [CrossRef] [PubMed] 
23. Offit, K.; Richardson, M.E.; Ceng, Q.Q.; Hampton, A.; Koduru, P.R.; Jhanwar, S.C.; Filippa, D.A.; Lieberman, P.H.; Clarkson, B.; Chaganti, R.S. Nonrandom chromosomal aberrations are associated with sites of tissue involvement in non-Hodgkin's lymphoma. Cancer Genet. Cytogenet. 1989, 37, 85-93. [CrossRef]

24. Rouze, A.; Boddaert, P.; Martin-Loeches, I.; Povoa, P.; Rodriguez, A.; Ramdane, N.; Salluh, J.; Houard, M.; Nseir, S. Impact of Chronic Obstructive Pulmonary Disease on Incidence, Microbiology and Outcome of Ventilator-Associated Lower Respiratory Tract Infections. Microorganisms 2020, 8, 165. [CrossRef] [PubMed]

25. Uzoamaka, M.; Ngozi, O.; Johnbull, O.S.; Martin, O. Bacterial Etiology of Lower Respiratory Tract Infections and Their Antimicrobial Susceptibility. Am. J. Med. Sci. 2017, 354, 471-475. [CrossRef] [PubMed]

26. Gales, A.C.; Sader, H.H.; Jones, R.N. Respiratory tract pathogens isolated from patients hospitalized with suspected pneumonia in Latin America: Frequency of occurrence and antimicrobial susceptibility profile: Results from the SENTRY Antimicrobial Surveillance Program (1997-2000). Diagn. Microbiol. Infect Dis. 2002, 44, 301-311. [CrossRef]

27. Lin, S.H.; Kuo, P.H.; Hsueh, P.R.; Yang, P.C.; Kuo, S.H. Sputum bacteriology in hospitalized patients with acute exacerbation of chronic obstructive pulmonary disease in Taiwan with an emphasis on Klebsiella pneumoniae and Pseudomonas aeruginosa. Respirology 2007, 12, 81-87. [CrossRef]

28. Khan, S.; Priti, S.; Ankit, S. Bacteria Etiological Agents Causing Lower Respiratory Tract Infections and Their Resistance Patterns. Iran. Biomed. J. 2015, 19, 240-246. [CrossRef]

29. Shah, M.U.; Mahmood, M.; Usman, J.; Kaleem, F.; Khalid, A. Gram negative organisms in community acquired respiratory tract infections. J. Microbiol. Infect. Dis. 2013, 3, 8-11. [CrossRef]

30. Xie, R.; Zhang, X.D.; Zhao, Q.; Peng, B.; Zheng, J. Analysis of global prevalence of antibiotic resistance in Acinetobacter baumannii infections disclosed a faster increase in OECD countries. Emerg. Microbes Infect. 2018, 7, 31. [CrossRef]

31. Adams, M.D.; Goglin, K.; Molyneaux, N.; Hujer, K.M.; Lavender, H.; Jamison, J.J.; MacDonald, I.J.; Martin, K.M.; Russo, T.; Campagnari, A.A.; et al. Comparative genome sequence analysis of multidrug-resistant Acinetobacter baumannii. J. Bacteriol. 2008, 190, 8053-8064. [CrossRef]

32. Mahillon, J.; Chandler, M. Insertion sequences. Microbiol. Mol. Biol. Rev. 1998, 62, 725-774. [CrossRef]

33. Franci, G.; Crudele, V.; Della Rocca, M.T.; Melardo, C.; Chianese, A.; Finamore, E.; Bencivenga, F.; Astorri, R.; Vitiello, M.; Galdiero, E.; et al. Epstein-Barr Virus Seroprevalence and Primary Infection at the University Hospital Luigi Vanvitelli of Naples from 2007 to 2017. Intervirology 2019, 62, 15-22. [CrossRef]

34. Falanga, A.; Del Genio, V.; Kaufman, E.A.; Zannella, C.; Franci, G.; Weck, M.; Galdiero, S. Engineering of Janus-Like Dendrimers with Peptides Derived from Glycoproteins of Herpes Simplex Virus Type 1: Toward a Versatile and Novel Antiviral Platform. Int. J. Mol. Sci. 2021, 22, 6488. [CrossRef]

35. Yayan, J.; Ghebremedhin, B.; Rasche, K. Antibiotic Resistance of Pseudomonas aeruginosa in Pneumonia at a Single University Hospital Center in Germany over a 10-Year Period. PLoS ONE 2015, 10, e0139836. [CrossRef]

36. Loomba, P.S.; Taneja, J.; Mishra, B. Methicillin and Vancomycin Resistant S. aureus in Hospitalized Patients. J. Glob. Infect. Dis. 2010, 2, 275-283. [CrossRef] [PubMed]

37. Pitz, A.M.; Yu, F.; Hermsen, E.D.; Rupp, M.E.; Fey, P.D.; Olsen, K.M. Vancomycin susceptibility trends and prevalence of heterogeneous vancomycin-intermediate Staphylococcus aureus in clinical methicillin-resistant S. aureus isolates. J. Clin. Microbiol. 2011, 49, 269-274. [CrossRef] [PubMed]

38. Pignataro, D.; Foglia, F.; Della Rocca, M.; Melardo, C.; Santella, B.; Folliero, V.; Shinde, S.; Pafundi, P.; Sasso, F.; Iovene, M. Methicillin-resistant Staphylococcus aureus: Epidemiology and antimicrobial susceptibility experiences from the University Hospital 'Luigi Vanvitelli'of Naples. Pathog. Glob. Health 2020, 114, 451-456. [CrossRef] [PubMed]

39. Petrillo, F.; Pignataro, D.; Lavano, M.A.; Santella, B.; Folliero, V.; Zannella, C.; Astarita, C.; Gagliano, C.; Franci, G.; Avitabile, T. Current Evidence on the Ocular Surface Microbiota and Related Diseases. Microorganisms 2020, 8, 1033. [CrossRef]

40. Petrillo, F.; Folliero, V.; Santella, B.; Franci, G.; Foglia, F.; Trotta, M.C.; Della Rocca, M.T.; Avitabile, T.; Gagliano, C.; Galdiero, M. Prevalence and Antibiotic Resistance Patterns of Ocular Bacterial Strains Isolated from Pediatric Patients in University Hospital of Campania "Luigi Vanvitelli," Naples, Italy. Int. J. Microbiol. 2020, 2020. [CrossRef]

41. Ahmed, S.M.; Jakribettu, R.P.; Meletath, S.K.; Arya, B.; Vpa, S. Lower Respiratory Tract Infections (LTRIs): An Insight into the Prevalence and the Antibiogram of the Gram Negative, Respiratory, Bacterial Agents. J. Clin. Diagn. Res. 2013, 7, 253-256. [CrossRef]

42. Prestinaci, F.; Pezzotti, P.; Pantosti, A. Antimicrobial resistance: A global multifaceted phenomenon. Pathog. Glob. Health 2015, 109, 309-318. [CrossRef]

43. Regha, I.; Sulekha, B. Bacteriological profile and antibiotic susceptibility patterns of lower respiratory tract infections in a tertiary care hospital, Central Kerala. Int. J. Med. Microbiol. Trop. Dis. 2018, 4, 186-190.

44. Scott, J.A.G. The global epidemiology of childhood pneumonia 20 years on. Bull. World Health Organ. 2008, 86, 494-496. [CrossRef] [PubMed]

45. Tchatchouang, S.; Nzouankeu, A.; Kenmoe, S.; Ngando, L.; Penlap, V.; Fonkoua, M.-C.; Pefura-Yone, E.-W.; Njouom, R. Bacterial Aetiologies of Lower Respiratory Tract Infections among Adults in Yaoundé, Cameroon. BioMed Res. Int. 2019, $2019,4834396$. [CrossRef] [PubMed]

46. Ullah, B.; Ahmed, S.; Shahariar, M.; Yesmine, S. Current trend of antibiotic resistance in lower respiratory tract infections (LRTIs): An experience in a teaching hospital in Bangladesh. Bangladesh Pharm. J. 2016, 19, 85-91. [CrossRef] 
47. Rossolini, G.; Mantengoli, E. Antimicrobial resistance in Europe and its potential impact on empirical therapy. Clin. Microbiol. Infect. 2008, 14, 2-8. [CrossRef]

48. Manyi-Loh, C.; Mamphweli, S.; Meyer, E.; Okoh, A. Antibiotic Use in Agriculture and Its Consequential Resistance in Environmental Sources: Potential Public Health Implications. Molecules 2018, 23, 795. [CrossRef] [PubMed]

49. Dalhoff, A. Global fluoroquinolone resistance epidemiology and implictions for clinical use. Interdiscip. Perspect. Infect. Dis. 2012, 2012. [CrossRef] [PubMed] 